\title{
Comportamiento de la repoblación natural de Pinus arizonica Engelm. bajo diferentes coberturas
}

\author{
Juan Manuel Chacón Sotelo ${ }^{1}$ \\ Alejandro Velázquez Martínez ${ }^{2}$ \\ Miguel Angel Musálem ${ }^{3}$
}

\begin{abstract}
RESUMEN
El manejo adecuado de las masas forestales, requiere del conocimiento preciso del número, ubicación y características específicas de los árboles remanentes después de una corta de repoblación. Para determinar el número de árboles a dejar en cortas de repoblación de árboles padres en un rodal natural de Pinus arizonica Engelm en Chihuahua, se evaluó la influencia de cinco grados de cobertura de dosel (64.5, $119.5,204.0,233.0$ y $345.0 \mathrm{~m}^{2} \mathrm{ha}^{-1}$ ) en la germinación, supervivencia y crecimiento inicial de plántulas, a los 6, 12, 18 y 34 meses después de aplicar la corta. La germinación fue mayor en condiciones de dosel abierto (64.50 $\mathrm{m}^{2}$ ha-1 de cobertura); sin embargo, en esta cobertura ocurrió la mayor mortandad de plántulas, en especial, inmediatamente después de ocurrida la germinación. El mayor crecimiento de los brinzales se observó entre las edades de 6 y 12 meses, en las coberturas más abiertas.
\end{abstract}

PALABRAS CLAVE:

Pinus arizonica, repoblación natural, árboles padres, germinación, crecimiento, supervivencia.

\begin{abstract}
The proper management of forest stands requieres knowledge about number, arrangement, and characteristics of seedtrees after the application of a reproduction method. In order to determine the number of seed-trees best suited in the seed-trees repopulation method in a Pinus arizonica Engelm. natural stand in Chihuahua state, the effect of five degrees of canopy closure $\left(64.5,119.5,204.0,233.0\right.$ y $\left.345.0 \mathrm{~m}^{2} \mathrm{ha}^{-1}\right)$ on seed germination, and survival and growth of seedlings was evaluated at 6,12 , 18 and 34 months after the application of final cut. Seed germination of the species was better in canopies of $64.50 \mathrm{~m}^{2} \mathrm{ha}^{-1}$; however, in this condition high mortality occurred, specially inmediately after seed germination. Greater seedling growth was observed between seedling ages 6 and 12 months under wide canopy openings.
\end{abstract}

KEY WORDS:

Pinus arizonica, natural regeneration, seed trees, germination, growth, survival.

1 Instituto Nacional de Investigaciones Forestales y Agropecuarias. Campo Experimental Madera. Madera, Chihuahua.

2 Programa Forestal. IRENAT. Colegio de Posgraduados. 56230. Montecillo, Edo. de México. México. alejvela@colpos.colpos.mx

3 Instituto Nacional de Investigaciones Forestales y Agropecuarias. Campo Experimental Valle de México. 56230. Chapingo, Edo. de México, México.

Manuscrito recibido para su publicación el 13 de mayo de 1998. 


\section{INTRODUCCION}

El método de repoblación de árboles padres, dentro de un sistema silvícola, ha resultado práctico y constituye una alternativa de bajo costo, para un gran número de especies, siempre y cuando exista la seguridad de un suministro adecuado de semilla. Sin embargo, la repoblación natural de la mayoría de las especies forestales bajo aprovechamiento, generalmente resulta incierta, debido al poco conocimiento que se tiene de la respuesta de las especies al ser tratadas con un método silvícola.

También se postula que el crecimiento de la repoblación natural es afectado durante el proceso de establecimiento, por el grado de sombra provocado por la copa de los árboles padres, y que después del establecimiento de las plántulas, la mortalidad ocurre, principalmente, durante los primeros meses después de la germinación. Por lo tanto, la intensidad de luz que llega al piso forestal mediante la apertura del dosel superior, propicia condiciones ambientales favorables para la repoblación natural.

En México, a partir de 1975 se viene aplicando de manera intensiva el método de repoblación de Arboles Padres; sin embargo, el número de árboles padres a dejar se determina de manera empírica, ya que en la mayoría de los casos no se tiene conocimiento de las características autoecológicas de las especies que determinan el grado de respuesta de los procesos que intervienen en el establecimiento de la repoblación natural (i.e. germinación y crecimiento).

Aunque para algunas especies forestales mexicanas como Abies religiosa Schl. et Cham., Pinus montezumae Lamb. $y$ Pinus hartwegii Lind. se han estudiado diversas condiciones ambientales en las cuales ocurre la repoblación natural bajo diferentes sistemas silvícolas (Manzanilla, 1974; Musálem, 1984; Velázquez et al.,
1985, 1986; Mas y González, 1990; González et al., 1991), la información de la respuesta a estos tratamientos aún no se precisa para otras especies de importancia económica.

Es conocido que dentro de su área de distribución, el Pinus arizonica presenta cierto potencial a repoblarse en forma natural; sin embargo, se desconocen aspectos silvícolas que lo condicionan. Por lo anterior, en este trabajo se tratan algunos aspectos asociados a su repoblación natural, se caracteriza la repoblación natural, desde su emergencia hasta los 34 meses de edad, con base a la germinación, el crecimiento en altura y la supervivencia, en función de diferentes grados de cobertura de los árboles padres, en la región de Ciudad Madera, Chihuahua.

\section{MATERIALES Y METODOS}

El presente estudio se basa en un experimento de largo plazo establecido en 1979 (Chacón, 1983). La descripción general del sitio y el establecimiento del ensayo en forma detallada se encuentra en el trabajo mencionado. Así mismo, se informa de los primeros resultados del establecimiento de la regeneración por tratamiento y en general del ensayo para las dos primeras mediciones, espaciadas seis meses, del año 1980, un año después de aplicada la corta de regeneración. Por lo anterior, en el presente trabajo, se enfatizan los análisis de las mediciones de supervivencia a largo plazo, y los efectos del dosel sobre el crecimiento de los brinzales, desde 1980 hasta 1983, aunque se toman en cuenta también los datos de 1980 del trabajo de Chacón relativo a la supervivencia de plántulas.

El sitio de estudio se ubicó en los bosques del Area Experimental Madera, localizada en la Sierra Madre Occidental, en la parte noroeste del estado de Chihuahua, a $29^{\circ} 08^{\prime} \mathrm{N}, 108^{\circ} 11^{\prime} \mathrm{O}$ y a $2245 \mathrm{~m}$ de altitud, donde el clima corresponde a 
templado sub-húmedo; en la estación meteorológica de Madera, Chihuahua, a $2090 \mathrm{~m}$ de altitud, la precipitación anual es $748 \mathrm{~mm}$; la temperatura media anual, 10.7 ${ }^{\circ} \mathrm{C}$; la media mínima, $5.0{ }^{\circ} \mathrm{C}$ y la media máxima $23{ }^{\circ} \mathrm{C}$. La temperatura mínima extrema es $22{ }^{\circ} \mathrm{C}$ (enero) y la máxima extrema $33^{\circ} \mathrm{C}$ (junio); el promedio anual de días con heladas y nevadas es de 175 y 8 días, respectivamente (Narváez, 1990). El $69 \%$ de la precipitación anual en esta zona ocurre en el verano y el restante $(31 \%)$ en invierno (Salmón, 1979).

La corta se aplicó a principios de 1979, en un rodal maduro uniespecífico de Pinus arizonica, con una edad promedio de 70 años, formando una estructura coetánea en manchones y con algunos claros en los cuales se observó, aunque escasa, cierta repoblación avanzada en etapa de brinzal.

El experimento se estableció en un diseño experimental de bloques al azar, con cinco tratamientos y tres repeticiones. Los tratamientos consistieron en cinco coberturas de dosel de los árboles padres: $64.5,119.5,204.0,233.0$ y $345.0 \mathrm{~m}^{2} \mathrm{ha}^{-1}$, correspondiendo a 4, 8, 12, 16 y 20 árboles por hectárea, respectivamente.

La cama semillera se preparó mediante la extracción de la trocería, con lo cual se provocó una remoción en las primeras capas del suelo (hojarasca y humus), poniendo al descubierto el horizonte mineral; posteriormente se apiló y quemó los desperdicios originados por la misma corta, más la vegetación arbustiva.

Se establecieron parcelas de $2,500 \mathrm{~m}^{2}$ (50x50 m),en cada una de las que se ubicaron cuatro sitios de muestreo circulares permanentes $\left(100 \mathrm{~m}^{2}\right)$, localizados a $25 \mathrm{~m}$ del centro de la parcela, en un ángulo de $45 /$.

La toma de datos, se efectuó a las edades de 2, 6, 12, 18, y 34 meses. En la primera medición, solamente se cuantificaron las plántulas germinadas; durante las mediciones posteriores, se consideró además, la altura de las plántulas.

Las variables analizadas fueron: a) número de plántulas registradas a la edad de 2 meses; b) supervivencia a la edad de $6,12,18$ y 34 meses y, c) altura promedio de las plántulas a las edades de 6, 12, 18 y 34 meses. Las evaluaciones estadísticas se practicaron por separado para cada una de las edades señaladas.

Para evaluar la supervivencia ocurrida en la población, se consideró la primera medición (octubre de 1980) como la edad inicial, a partir de la cual se estimó el número de plantas vivas por hectárea entre cada periodo de evaluación $(6,12,18$, y 34 meses). Con ello, se obtuvo el porcentaje de plantas vivas, mismo que se utilizó para efectuar los análisis de varianza para cada edad. Para comparar las medias de los tratamientos se aplicó la prueba de Tukey.

\section{RESULTADOS Y DISCUSION}

La germinación de $P$. arizonica fue mayor (27,541 plántulas/ha) donde la cobertura fue menor (64.50 $\left.\mathrm{m}^{2} \mathrm{ha}^{-1}\right)$; contrariamente, en la cobertura mayor (345.0 $\left.\mathrm{m}^{2} \mathrm{ha}^{-1}\right)$, la emergencia de las plántulas tuvo una respuesta significativamente menor $(18,620$ plántulas/ha) (Tabla 1). Lo anterior indica que las semillas de esta especie requieren condiciones de sustrato de germinación en condiciones de mayor iluminación, que resulta en mayor temperatura para la germinación (Musálem, 1984; Velázquez et al., 1986).

Por el contrario, con respecto a la supervivencia, las condiciones con cobertura mayor que $204 \mathrm{~m}^{2} \mathrm{ha}^{-1}$ provocaron menor insolación, lo que protege a las plántulas recién germinadas durante sus primeros seis meses de vida (Tabla 1). Más tarde, sin embargo, a las edades de 12, 18 y 34 meses, este efecto 
de protección provocado por los grados de cobertura pasa a ser secundario (Tabla 1).

Fue notable la reducción general del número de plántulas a medida que crecían. La reducción del número de individuos es mayor, en los primeros meses de vida (6 y 12 meses de edad); por ejemplo, en la cobertura de $64.5 \mathrm{~m}^{2}$ ha $^{-1}$, cuya germinación fue superior a los 27,500 individuos, a la edad de 12 meses, la población disminuyó a 11,250 plantas vivas por unidad de superficie, llegando al final de los 34 meses, a sólo 5,175 individuos. Asimismo, la cobertura de $345.0 \mathrm{~m}^{2} \mathrm{ha}^{-1}$, con una población original de 18,620 individuos por hectárea, se redujo a 15575 a la edad de 12 meses y concluyó con 10,050 individuos al cabo de los 34 meses (Tabla 1). Esto indica una reducción porcentual en el número de individuos superior en las áreas más abiertas comparadas con las más cerradas por las copas de los árboles padres.

Tomando en cuenta que en el establecimiento de un nuevo rodal, la supervivencia del renuevo representa el principal objetivo en las primeras etapas del establecimiento (Daniel et al., 1982; Minore,1986; Smith, 1986), la cobertura de $345.0 \mathrm{~m}^{2} \mathrm{ha}^{-1}$ fue la que presentó el mayor número de plántulas a los 34 meses y el mayor porcentaje de supervivencia (53\%) respecto a las plántulas germinadas (Tabla $1)$.

Con respecto al crecimiento de los brinzales, éstos alcanzaron las menores alturas, durante los primeros meses de vida, donde la cobertura ocasiona una mayor interferencia de luz. Este resultado indica que la protección necesaria para aumentar la supervivencia, con el paso del tiempo, tiene un efecto negativo para el crecimiento de la repoblación. Aún cuando los valores de altura a los 34 meses no son estadísticamente significativos, los valores de altura comienzan a indicar la necesidad de liberar a las nuevas plántulas de la competencia de las copas de los árboles padres, atendiendo sobre todo, al característico crecimiento cespitoso de los brinzales, que iniciarán el crecimiento acelerado en altura una vez que se liberen de los árboles padres (Tabla 2).

En general, se puede establecer que las aperturas de dosel mayores favorecen la germinación y que la exposición directa al sol disminuye la sobrevivencia (Musálem, 1984; Velázquez et al.,1986), sobre todo en la primeras etapas de crecimiento de las plántulas, aunque al final de casi tres años las diferencias tienden a desaparecer, las tasas de reducción de número de individuos es mayor en las áreas más expuestas. Por otro lado, las condiciones de crecimiento de apertura más abierta permiten un desarrollo en altura más grande en el primer año de vida de los brinzales, las diferencias también comienzan a desaparecer al final de los tres años, aunque se comienza a observar un menor crecimiento bajo las coberturas más cerradas, lo que sugiere para un buen manejo silvícola de la especie, la edad de inicio de la eliminación gradual del dosel de árboles para continuar asegurando la supervivencia inicial y el crecimiento óptimo de los brinzales.

Los resultados anteriores son consistentes con los de otras especies de tolerancia similar como P. ponderosa Laws., $P$. montezumae Lamb. y P. hartwegii Lindl., donde se recomienda la liquidación gradual del dosel para permitir una cierta protección a la repoblación durante las primeras etapas de establecimiento $y$, posteriormente su liberación para favorecer su crecimiento (Schubert, 1974; Musálem,1984; Velázquez et al. 1985, 1986).

Esto significa que para esta especie, en esta zona, sería recomendable dejar el número máximo de árboles semilleros por hectárea, y una vez establecida la repoblación natural, liberar la masa por lo menos hasta después de tres años de aplicada la corta final, con lo cual se puede garantizar un buen nivel de supervivencia y tasas de crecimiento. 
Tabla 1. Germinación y supervivencia (plántulas por hectárea) del renuevo de Pinus arizonica Engelm., en cinco grados de cobertura de árboles padres, en Madera, Chihuahua.

\begin{tabular}{||c|c|c|c|c|c||}
\hline \multirow{2}{*}{ COBERTURA } & \multicolumn{5}{|c||}{ EDAD (meses) } \\
\cline { 2 - 6 } & 2 & 6 & 12 & 18 & 34 \\
\hline Germinación & \multicolumn{5}{|c||}{ Supervivencia } \\
\hline 64.5 & $27,541 \mathrm{a}$ & $10,075 \mathrm{a}$ & $11,250 \mathrm{a}$ & $9,475 \mathrm{a}$ & $5,175 \mathrm{a}$ \\
119.5 & $22,783 \mathrm{ab}$ & $10,650 \mathrm{a}$ & $13,425 \mathrm{a}$ & $11,675 \mathrm{a}$ & $7,925 \mathrm{a}$ \\
204.0 & $23,250 \mathrm{ab}$ & $11,600 \mathrm{ab}$ & $13,450 \mathrm{a}$ & $10,925 \mathrm{a}$ & $7,200 \mathrm{a}$ \\
233.0 & $21,191 \mathrm{ab}$ & $13,575 \mathrm{ab}$ & $14,200 \mathrm{a}$ & $10,600 \mathrm{a}$ & $7,300 \mathrm{a}$ \\
345.0 & $18,620 \mathrm{~b}$ & $15,725 \mathrm{~b}$ & $15,575 \mathrm{a}$ & $14,475 \mathrm{a}$ & $10,050 \mathrm{a}$ \\
\hline
\end{tabular}

Dentro de columnas, los valores con la misma letra no son diferentes (Tukey, 0.05).

Tabla 2. Altura (cm) a diferentes edades de brinzales de Pinus arizonica Engelm., en cinco grados de cobertura de árboles padres, en Madera, Chihuahua.

\begin{tabular}{||c|c|c|c|c||}
\hline \multirow{2}{*}{$\begin{array}{c}\text { COBERTURA } \\
\mathrm{m}^{2} \text { ha }^{-1}\end{array}$} & \multicolumn{4}{|c||}{ EDAD (meses) } \\
\cline { 2 - 5 } & 6 & 12 & 18 & 34 \\
\hline 64.5 & $6.6 \mathrm{a}$ & $7.7 \mathrm{ab}$ & $11.0 \mathrm{a}$ & $16.7 \mathrm{a}$ \\
119.5 & $6.0 \mathrm{ab}$ & $8.4 \mathrm{a}$ & $12.2 \mathrm{a}$ & $18.3 \mathrm{a}$ \\
204.0 & $5.8 \mathrm{ab}$ & $6.8 \mathrm{ab}$ & $9.3 \mathrm{a}$ & $17.3 \mathrm{a}$ \\
233.0 & $5.7 \mathrm{ab}$ & $7.1 \mathrm{ab}$ & $9.6 \mathrm{a}$ & $12.3 \mathrm{a}$ \\
345.0 & $5.1 \mathrm{~b}$ & $5.5 \mathrm{~b}$ & $7.4 \mathrm{a}$ & $12.0 \mathrm{a}$ \\
\hline
\end{tabular}

Dentro de columnas, los valores con la misma letra no son diferentes (Tukey, 0.05).

\section{CONCLUSIONES}

1. En el menor grado de cobertura de árboles padres $\left(64.5 \mathrm{~m}^{2} \mathrm{ha}^{-1}\right)$, se presentó la mayor emergencia de plántulas; sin embargo, fue la que presentó la mayor mortandad en la etapa post-germinativa.

2. La cobertura de árboles padres de $345.0 \mathrm{~m}^{2} \mathrm{ha}^{-1}$, representa la mejor condición para lograr una mayor supervivencia (53\%), al final de los 34 meses de edad del renuevo.
3. La estabilización de la reducción del número de plántulas por hectárea se alcanzó a la edad de 18 meses, lo cual puede considerarse como el tiempo necesario para el establecimiento completo de la repoblación natural.

4. El crecimiento en altura en las edades de 6 y 12 meses fue mayor en las coberturas menores (64.5 y $119.5 \mathrm{~m}^{2}$ ha $\left.^{-1}\right)$; aunque tiende a igualarse a los 18 y 34 meses, se aprecia un crecimiento mayor en las coberturas menores, como al inicio del establecimiento. 


\section{REFERENCIAS}

Chacón, J.C. 1983. Regeneración mediante árboles padres de Pinus arizonica. Ciencia Forestal 42(8):3-20.

Daniel, P.W., U.E. Helms y F.S. Baker. 1982. Principios de Silvicultura. Elizondo M., R. (trad.). McGraw-Hill. México. 492 p.

González G., M.J., M.A. Musálem, G. Zárate y A. Velázquez M. 1991. Estudio de la germinación del oyamel [Abies religiosa (H.B.K.) Schl. et Cham.] en condiciones naturales en Zoquiapan, México. Revista Chapingo 15(75): 59-66.

Manzanilla, H. 1974 . Investigaciones Epidométricas y Silvícolas de Abies religiosa. Secretaría de Agricultura y Recursos Hidraúlicos. México, D.F. $345 \mathrm{p}$.

Mas P., J. y P.A. González. 1990. El Sitio permanente de experimentación forestal "La Nieve" a 27 años de su establecimiento. Centro de Investigaciones Forestales y Agropecuarias del Pacífico. Secretaría de Agricultura y Recursos Hidráulicos. México. $45 \mathrm{p}$.

Minore, D. 1986. Germination, survival and early growth of conifer seedlings in two habitat types. U.S. For. Serv. Res. Paper. PNW-348. 25 p.

Musálem, M.A. 1984. Effect of environmental factors on regeneration of Pinus montezumae Lamb., in a temperate forest of México. Ph. D. Dissertation. Yale University. New Haven, Conn. EUA. 262 p.
Narváez F., R. 1990. El suelo en relación con los tipos de vegetación y su productividad en el Area Experimental Forestal Madera, Chih. Tesis de Maestría en Ciencias. Universidad Nacional Autónoma de México. México. 107 p.

Salmón M., J. 1979. Influencia de la humedad invernal en el crecimiento en altura de los pinos en el estado de Chihuahua. In. La Investigación Forestal en los Organismos Descentralizados. Pub. Esp. No. 15. Instituto Nacional de Investigaciones Forestales. México, D.F. 33 p.

Schubert, G.H. 1974. Silviculture of southwestern ponderosa pine: the status of our knowledge. U.S. Forest. Service. Gen. Tech. Rep. RM-123. 71 p.

Smith, D.M. 1986. The Practice of Silviculture. 8th ed. John Wiley \& Sons, Inc. New York, EUA. 527 p.

Velázquez M., A., M.R. Keyes y G. Zárate 1985. Influencia de la intercepción de la luz solar en el crecimiento de la regeneración artificial de Pinus hartwegii Lindl. In: Memoria de la III Reunión Nacional sobre Plantaciones Forestales. Pub. Esp. No. 48. Secretaría de Agricultura y Recursos Hidraúlicos. México. pp: 579-594.

Velázquez M., A., M.A. Musálem, M.R. Keyes y G.L. Zárate. 1986. Influencia del tratamiento al suelo y la condición de apertura del dosel en el establecimiento inicial de la regeneración natural de Pinus hartwegii Lindl. Agrociencia 64: 147170. 